\title{
New hope: the emerging role of 5-hydroxymethylcytosine in mental health and disease
}

\begin{abstract}
Historically biomedical research has examined genetic influences on mental health but these approaches have been limited, likely due to the broad heritability of brain-related disorders (e.g., 30-90\%). Epigenetic modifications, such as DNA methylation, are environmentally sensitive mechanisms that may play a role in the origins and progression of mental illness. Recently, genome-wide disruptions of 5 -hydroxymethylcytosine $(5 \mathrm{hmC})$ were associated with the development of early and late onset mental illnesses such as autism and Alzheimer's disease, bringing new hope to the field of psychiatry. Here, we review the recent links of $5 \mathrm{hmC}$ to mental illness and discuss several putative functions of $5 \mathrm{hmC}$ in the context of its promising clinical relevance.
\end{abstract}

First draft submitted: 24 February 2016; Accepted for publication: 28 April 2016; Published online: 14 July 2016

Keywords: 5 -hydroxymethylcytosine $\bullet$ DNA methylation $\bullet$ epigenetics $\bullet$ mental illness

A fundamental problem in psychiatry is that diagnoses are based on subjective measures that are primarily acquired through behavioral assessments. Modern genetics has held out hope that one could identify new genetic markers of mental illness early in childhood that could aid the subjective measures of behavior used today. New laboratory tests for these markers would then indicate to psychiatrists the most promising drug for each person or direct pharmaceutical companies to more specific personalized drug development. However, even though complete mapping of the human genome was announced in 2003, the genetic approach has had little impact on improving the lives of individuals struggling with mental illness. This outcome is partially caused by the broad range of heritability in mental illness (i.e., 30-90\%) but also is due to a failure in molecular psychiatry that has overlooked a crucial molecular step that causes variations in the expression of key genes in the brain. Past approaches have largely ignored how environmental stimuli can affect the unique amount of gene expression in the brain of each person. Thus, the use of this personalized information provides new hope that one day it will be possible to develop a precise molecular-based treatment, which will change the way mental health is diagnosed and treated today.

Epigenetic mechanisms are environmentally sensitive molecular modifications that are heritable but do not change the underlying DNA sequence. Much effort has been put forth toward understanding two specific epigenetic mechanisms: histone modifications, which alter gene expression through the restructuring of chromatin proteins, and DNA methylation, which modulate gene expression through the addition of a methyl group to specific sites within the DNA sequence. The most studied epigenetic mechanism in the mammalian genome is DNA methylation, which is the addition of a methyl group on the fifth carbon of cytosine (e.g., 5-methylcytosine [5mC]). This DNA mark functions in genomic imprinting,
Andy Madrid ${ }^{1,2}$, Ligia A Papale ${ }^{1}$ \& Reid S Alisch ${ }^{*, 1}$ 'Department of Psychiatry, University of Wisconsin, Madison, WI 53719, USA

${ }^{2}$ Neuroscience training program, University of Wisconsin, Madison, WI 53719, USA

*Author for correspondence:

Tel.: +1 6082628430

Fax: +1 6082611103

alisch@wisc.edu 
X-chromosome inactivation, chromatin structure and gene silencing [1-5]. For a more definitive and technical perspective of $5 \mathrm{mC}$, please refer to Box 1 . Human studies also support a role for $5 \mathrm{mC}$ in the development of mental pathologies including bipolar disorder, schizophrenia and major depressive disorder, often resulting in concomitant changes in gene expression [6-10]. It was recently shown that $5 \mathrm{mC}$ can be oxidized to 5-hydroxymethylcytosine $(5 \mathrm{hmC})$ and that this modification is environmental sensitive [11], stable [12] and highly enriched in the brain [13-17]. While the molecular role(s) of $5 \mathrm{hmC}$ remain elusive, primarily due to the dearth of $5 \mathrm{hmC}$ functional studies, its unique enrichment in the brain [18] leads many to believe that $5 \mathrm{hmC}$ functions are independent from those of $5 \mathrm{mC}$, and that $5 \mathrm{hmC}$ may be a critical molecular building block in mental health that is disrupted in disease. For a more definitive and technical perspective of $5 \mathrm{hmC}$, please refer to Box 1. It is noteworthy that traditional DNA methylation detection methods utilizing sodium bisulfite treatment cannot distinguish between the methylated and hydroxymethylated forms of cytosine, meaning that past studies that utilized such methods report a composite of $5 \mathrm{mC}$ and $5 \mathrm{hmC}$ but have attributed any findings solely to $5 \mathrm{mC}$. This review will provide substantial evidence to support the independent importance of $5 \mathrm{hmC}$ in mental health and the development of mental illness and present several putative molecular functions of $5 \mathrm{hmC}$ that may shed light on its promising clinical relevance. With recent findings implicating a unique role for $5 \mathrm{hmC}$ in developmental brain disorders, $5 \mathrm{hmC}$ brings a new frontier to the field of psychiatry.

\section{Development, aging \& neurodegeneration}

$5 \mathrm{hmC}$ is essential for proper mammalian development, beginning with an early role in the distinction between embryonic stem cells (ESCs) and neural progenitor cells (NPCs) [33]. Here, the transition from ESC to NPC requires a genome-wide reduction of $5 \mathrm{hmC}$, which is facilitated by a dramatic change in the expression of the modulatory proteins required for oxidizing $5 \mathrm{mC}$ to $5 \mathrm{hmC}$ (TET1-3; Box 1) [34]. These findings link $5 \mathrm{hmC}$ and TET expression with ESC differentiation and suggest that aberrant $5 \mathrm{hmC}$ levels may impact neurogenesis. Consistent with this link, embryos completely lacking TET1 and TET3 experience lower $5 \mathrm{hmC}$ and higher $5 \mathrm{mC}$ levels, which results in more variable expression of important neurodevelopmental genes including those in the cholesterol biosynthesis pathway that lead to the development of holoprosencephaly, a cephalic disorder characterized by the failure of the two brain hemispheres to properly form [35]. To our knowledge, this is the only study that has been able to link deficits of $5 \mathrm{hmC}$ with phenotypic alterations in embryos. While these studies demonstrate a role

Box 1. DNA methylation.

$5 \mathrm{mC}$

- $5 \mathrm{mC}$ is a modified version of cytosine that involves the addition of a methyl group to the fifth carbon of cytosine and is nearly exclusive at CpG dinucleotide sites. This modification is catalyzed by DNMTs, which utilize S-adenosyl-L-methionine as the methyl donor [19]. Three active DNMTs have been identified in mammals and they have distinct functions. DNMT3a and DNMT3b have an affinity toward unmethylated CpG sites, while DNMT1 preserves methylation, as it shows preference toward hemimethylated $\mathrm{CpG}$ sites [20]. While CpG rich-regions, known as CpG islands, and gene promoters have a significant reduction in $5 \mathrm{mC}$ levels, the $\mathrm{X}$ chromosome has an overabundance of $5 \mathrm{mC}[21,22] .5 \mathrm{mC}$ regulates processes such as gene silencing, X-chromosome inactivation, genomic imprinting and chromatin structure [23]. The 'gold-standard' in the detection of $5 \mathrm{mC}$ is sodium bisulfite sequencing, which converts unmethylated cytosine to uracil that are read as thymine following PCR [24]

$5 \mathrm{hmC}$

- $5 \mathrm{hmC}$ is a modified version of cytosine that involves the addition of a hydroxy group to the methyl group of $5 \mathrm{mC}$. This modification is catalyzed by the TET family of enzymes [25]. An approximately tenfold abundance of $5 \mathrm{hmC}$ is seen in brain tissues, other tissues of the central nervous system and embryonic stem cells, compared with peripheral tissue [26]. 5hmC levels are reduced in CpG islands and intronic sequences, but are enriched in gene promoters, the 5'-UTR, exons, and distal regulatory regions [26]. Notably, mammals have a disproportionate reduction of $5 \mathrm{hmC}$ on chromosome 18 and the $\mathrm{X}$ chromosome [27]. While an increase in transcription levels has been correlated with $5 \mathrm{hmC}$ enrichment [26], alternatively spliced transcripts display a reduced $5 \mathrm{hmC}$ on excised exons as compared with constitutive exons, suggesting that $5 \mathrm{hmC}$ may be playing a role in the production of isoform transcripts [28]. Neuronal- and synapse-related genes have a significant increase in $5 \mathrm{hmC}$ levels [28]. The function of $5 \mathrm{hmC}$ has yet to be discovered, yet several putative functions are discussed in this review, including the regulation of transcription factor binding [29], gender-specific development [30] and transcript diversity through interactions with the spliceosome [31]. Since traditional sodium bisulfite sequencing cannot distinguish between $5 \mathrm{hmC}$ and $5 \mathrm{mC}$, Tet1-assisted bisulfite sequencing has become an established method for $5 \mathrm{hmC}$ detection [32]

5hmc: 5-hydroxymethylcytosine; 5mc: 5-methylcytosine. 
for $5 \mathrm{hmC}$ in neurodevelopment during embryogenesis, $5 \mathrm{hmC}$ continues to be abundant postnatally and undergoes age-dependent accumulation throughout early life development and into adulthood [36-38]. However, environmental stimuli, such as caloric restriction, can alter this age-associated accumulation of $5 \mathrm{hmC}$, as caloric restricted mice showed an age-dependent reduction of $5 \mathrm{hmC}$ in hippocampal and cerebellar tissue [39]. Together, these findings suggest that neurodevelopmental age and environment strongly influence $5 \mathrm{hmC}$ dynamics in the brain, which in turn can play a modulatory role in the expression of key genes involved in neurodevelopment.

The emerging link between $5 \mathrm{hmC}$ and brain development, and its accumulation with age, have led researchers to examine this epigenetic mark in neurodegenerative diseases [40]. Indeed, age-associated genes that acquire $5 \mathrm{hmC}$ are associated with pathways related to neurodegenerative diseases [25]. For example, depletion of $5 \mathrm{hmC}$ was found in the hippocampus, cerebellum and entorhinal cortex of patients suffering from Alzheimer's disease (AD) [41,42], a type of dementia that usually develops slowly and gets worse over time. On the other hand, enrichment of $5 \mathrm{hmC}$ in the frontal and midtemporal gyrus was positively correlated with hallmarks of $\mathrm{AD}$, including neurofibrillary tangles (NFT), amyloid beta and ubiquitin load [43]. Together, these findings suggest that $5 \mathrm{hmC}$ may be driving the primary molecular components of $\mathrm{AD}$ progression within distinct regions of the brain. Notably, AD-associated levels of $5 \mathrm{hmC}$ can be detected at preclinical stages as well as at later stages of AD [44], indicating that $5 \mathrm{hmC}$ may act as a viable biomarker of $\mathrm{AD}$ onset and progression. However, studies employing nuclear labeling or ELISA were unable to find significant alterations in $5 \mathrm{hmC}$ associated with $\mathrm{AD}$ [45], suggesting that higher resolution methods are required to identify AD-associated changes in $5 \mathrm{hmC}$. Connections between $5 \mathrm{hmC}$ and other neurodegenerative disorders including Huntington's, ataxia-telangiectasia and Fragile X-associated tremor/ataxia syndrome have recently surfaced and we will describe the link to $5 \mathrm{hmC}$ of each of these disorders below.

Huntington's disease (HD) is characterized by deficits in cognitive, psychiatric and motor stability that is typically caused by a CAG trinucleotide expansion in the HTT gene [46]. In addition to the mutation in the HTT gene, previous studies have suggested a role for epigenetic mechanisms in HD, such as altered histone modifications [47] and DNA methylation of a locus closely linked to the HTT gene known as DS495 [48]. These epigenetic connections led to the recent investigation of the genome-wide distribution of $5 \mathrm{hmC}$ in a mouse model of HD, which found a deficiency of
$5 \mathrm{hmC}$ in the mouse striatum and cortex tissues [49]. This altered distribution of $5 \mathrm{hmC}$ was generally associated with pathways involved in neuronal development/differentiation, axonal guidance and neuronal function/survival. A specific example included a $5 \mathrm{hmC}$ reduction on the $A D O R A 2 A$ gene, a G-proteincoupled receptor that decreases its expression in HD patients, which revealed that $5 \mathrm{hmC}$ may play a role in the pathological expression of ADORA2A found in HD patients [50]. Together, these results implicate the loss of $5 \mathrm{hmC}$ as a potential molecular mechanism underlying the cause of neuronal cell death associated with HD.

Ataxia-telangiectasia, a devastating neurodegenerative disorder that shows high cell specificity in its development, is caused by a mutation in the ATM gene [51]. Despite this monogenic etiology, alterations in $5 \mathrm{hmC}$ were recently found in repeated sequences and regulatory elements of DNA from ATM-deficient Purkinje cells from mice and humans [52]. This $5 \mathrm{hmC}$ distribution is likely the result of TET1 responding to DNA damage, which is a hallmark of $A T M$-deficient neuronal cells. To test whether TET1 activity is related to Purkinje cell degeneration and behavioral deficits in mice, adenoviral particles encoding either human wild-type TET1 (TET1-WT) or kinase dead mutant TET1 (TET-KD) were infected into wild-type and ATM-deficient mouse cerebellum slices. Cerebellum samples lacking TET1 expression that were infected with TET1-WT showed no activation of CASPASE-3, a cell death marker, while infection with TET1-KD activated CASPASE-3. These findings suggest that TET1-mediated $5 \mathrm{hmC}$ production is essential for Purkinje cell viability and the prevention of ataxia-telangiectasia-like symptoms in mice, supporting the concept that vulnerability to neurodegeneration is linked to aberrant changes of $5 \mathrm{hmC}$ in neuronal cells.

Fragile X-associated tremor/ataxia syndrome (FXTAS), a late-onset neurodegenerative disorder, is one of the characterized Fragile-X disorders that are caused by a CGG expansion in the 5'UTR of the FMR1 gene [53,54]. Genome-wide analysis of $5 \mathrm{hmC}$ in the cerebellum of a FXTAS mouse model (rCGG mice) revealed an overall reduction in $5 \mathrm{hmC}$ at 16 weeks of age when compared with age-matched controls [55]. Despite the overall reduction of $5 \mathrm{hmC}$, these mice have an increase of $5 \mathrm{hmC}$ in repetitive sequences as well as in cerebellum-specific enhancers. Differential $5 \mathrm{hmC}$ between the rCGG and control mice were predominantly found in transcription factor (TF) binding sites that are located in genes essential for neuronal development. Finally, ribosomal profiling revealed that the differential $5 \mathrm{hmC}$-associated genes often exhibited altered ribosomal processing in the rCGG mice, 
suggesting that $5 \mathrm{hmC}$ may somehow influence translational changes. In summary, this study links $5 \mathrm{hmC}$ to the etiology of FXTAS and implicates a role for $5 \mathrm{hmC}$ in TF binding and in regulating ribosomal processing of mature RNA transcripts.

Despite that the above disease associated disruptions in $5 \mathrm{hmC}$ represent changes across age; these examples clearly demonstrate a role for $5 \mathrm{hmC}$ in proper development and in the onset and progression of neurodegenerative diseases. These developmental and neurodegenerative disease-associated changes in $5 \mathrm{hmC}$ often arise within distinct cell-types and brain regions, supporting cell and tissue-specific development of these diseases. Since these studies have been largely descriptive, it will be imperative to determine the functional mechanism(s) played by $5 \mathrm{hmC}$ if we are to modify it toward healthy outcomes.

\section{Sensitivity to environmental stimuli}

The abundance and distribution of $5 \mathrm{hmC}$ is sensitive to environmental stimuli. Mice exposed to enriched environment show reduced $5 \mathrm{hmC}$ abundance in the hippocampus primarily on genes involved in axon guidance. These alterations were also associated with increased learning and memory, suggesting that environmental enrichment might modulate the dynamics of $5 \mathrm{hmC}$ in the hippocampus and contribute to improved learning and memory [56]. In contrast, our group found increased $5 \mathrm{hmC}$ abundance on the glucocorticoid receptor gene $(\mathrm{Nr} 3 \mathrm{cl})$ in the hippocampus of mice stressed for $30 \mathrm{~min}$ and allowed to recover for $1 \mathrm{~h}$ [57]. Genome-wide $5 \mathrm{hmC}$ analysis of these same mice revealed that short-term stress induced genomewide disruptions of $5 \mathrm{hmC}$ and confirmed an overall increase in $5 \mathrm{hmC}$ following stress. Similar to the FXTAS mouse model described above, altered $5 \mathrm{hmC}$ was found in several TF binding sites of genes. Here these genes also were differentially expressed and have known roles in neurogenesis and neurological activities [29], suggesting that in response to stress the function of $5 \mathrm{hmC}$ may be to influence TF binding to provide appropriate levels of gene expression needed to cope with the stress. The fact that $5 \mathrm{hmC}$ changes were found within $1 \mathrm{~h}$ following a short stress highlights the potential for rapid changes of $5 \mathrm{hmC}$ within the brain. It will be interesting to examine the long-term effects of short stress (i.e., more than $1 \mathrm{~h}$ after exposure) or how chronic stress alters $5 \mathrm{hmC}$ levels. Many believe $5 \mathrm{hmC}$ to be important in long-term consequences of mental health, yet this study indicates that alterations in $5 \mathrm{hmC}$ can occur rapidly and may impact the expression of key genes related to the origins of mental illnesses. While environmental stressors have been shown to alter gene expression [58], a definitive molecu- lar culprit has been slow to emerge, perhaps $5 \mathrm{hmC}$ is a promising candidate.

Environmental stimuli affects brain regions other than the hippocampus. For example, mice exposed to repeated administrations of cocaine have increased $5 \mathrm{hmC}$ in the nucleus accumbens, primarily in coding regions and enhancer sequences of genes involved in drug addiction [31]. Notably, $5 \mathrm{hmC}$ changes persisted for a minimum of 1 month after cocaine exposure in only a small subset of loci, suggesting that these epigenetic changes are largely reversible. In addition, modulation of $5 \mathrm{hmC}$ may also mediate behavioral adaptations. For example, fear extinction, a form of reverse learning, results in dramatic $5 \mathrm{hmC}$ changes in the prefrontal cortex of mice [59]. These studies also supported unique molecular roles for the TET enzymes, as TET3, but not TET1 mediated the increased gene expression that was associated with rapid behavioral adaptation. Interestingly, another group found that mice lacking the expression of Tet 1 exhibited impaired memory extinction, coupled with long-term synaptic depression and down-regulation of neuronal activityrelated genes [60]. Thus, while TET3 may solely facilitate the accumulation of $5 \mathrm{hmC}$ in the prefrontal cortex of mice during rapid behavior adaptation in response to fear, TET1 governs alterations in $5 \mathrm{hmC}$ on synaptic plasticity genes during behavioral adaptation in response to stressful environmental exposures.

Taken together, these studies open up the possibility that $5 \mathrm{hmC}$ may function in the development of environmentally sensitive neuronal dysfunction. It will be of great interest to investigate the role of each TET enzyme coupled with the rapid and stable dynamics of $5 \mathrm{hmC}$ at different developmental time points to understand its role in synaptic plasticity, neuronal development, the maintenance of mental health and the onset of mental illness.

\section{Early life adversity, $5 \mathrm{hmC} \&$ the origins of psychiatric disorders}

Several studies indicate that early life experiences have a profound impact on brain development and subsequent adult behavior [61-64]. Recent evidence indicates that the epigenome is a potential molecular mechanism governing the long-lasting effects of early life stress on brain and behavior. One such example involves rhesus macaques that were deprived of early life maternal interactions. As adults, these monkeys have altered $5 \mathrm{hmC}$ in the prefrontal cortex on promoters of genes related to neurological functions and psychological disorders (e.g., DRD3, 5-HTT, and GABRA2) [65]. Since these $5 \mathrm{hmC}$ disruptions were detected during adulthood, early life changes in $5 \mathrm{hmC}$ also can be stable throughout development and may represent 
the origins of developmental brain disorders such as schizophrenia, bipolar disorder and autism.

\section{Schizophrenia, bipolar disorder \& major depressive disorder}

Schizophrenia (SCZ) and bipolar disorder (BD) are psychiatric disorders with shared and distinct clinical and genetic features. In both disorders, stressful events increase the risk for onset and relapse mainly through the dysregulation of the hypothalamuspituitary-adrenal (HPA) axis. Although many genes are linked to HPA dysfunction, the majority of SCZ and $\mathrm{BD}$ cases cannot be explained by genetics alone and the epigenome likely has a role in the etiology of these disorders. Consistent with this hypothesis, rodent models exposed to prenatal stress exhibit longlasting neurological, endocrinological and behavioral changes that are thought to mirror the development of SCZ [66]. These models show increased DNA methylation $(5 \mathrm{mC}+5 \mathrm{hmC})$ in GABAergic interneurons primarily in CpG-rich regions of GABAergic genes [67,68]. Together, these studies demonstrated that prenatal stressors can alter DNA methylation levels in the brain and implicate a role for DNA methylation in the origins of SCZ and mood disorders [69]. In humans, an independent role for $5 \mathrm{hmC}$ in SCZ and BD patients is characterized by increased $5 \mathrm{hmC}$ abundance and TET1 expression, but not TET2 or TET3, in the inferior parietal lobule (IPL) [70]. Remarkably, TET1 was not altered in the cerebellum of these patients, suggesting that $5 \mathrm{hmC}$ may be involved in the development of psychosis through the inferior parietal lobule, but not the cerebellum, perhaps shedding light on the tissuespecific development of SCZ and BD. The increases of $5 \mathrm{hmC}$ in these patients were associated with reduced expression of biologically relevant genes including GAD67 and APOBEC3A, which is an enzyme with critical roles in the active DNA demethylation pathway. In contrast, patients only suffering from severe major depressive disorder have significantly decreased levels of $5 \mathrm{hmC}$ [71], suggesting that $5 \mathrm{hmC}$ has unique functions among closely related brain disorders. Together, these findings suggest a common etiology in psychosis, one that includes early life adversity and genome-wide changes in $5 \mathrm{hmC}$.

\section{Autism}

The autism spectrum disorders (ASD) encompass a broad range of behaviorally related and neurodevelopmental disorders with a high prevalence in children. Notably, only $\sim 20 \%$ of ASD cases show a genetic etiology $[72,73]$. Prenatal factors shown to increase the risk of ASD in offspring [74] include environmental influences such as multiple births, in vitro fertilization and parental exposure to common drug treatments (e.g., antiepileptic drugs [e.g., valporate] or folic acid) [75]. Together, these findings effectively open the door for contributions from epigenetic modifications such as $5 \mathrm{hmC}$ to have an underlying role in the ASD etiology. Consistent with this hypothesis, developmentally specific changes in $5 \mathrm{hmC}$ are highly enriched in known autism genes [76]. Moreover, we recently profiled $5 \mathrm{hmC}$ in a mouse model of autism that has behavioral and molecular alterations such as deficits in social interactions and GABAergic signaling [77,78]. This study revealed that this mouse model harbored differential $5 \mathrm{hmC}$ on a remarkable number of established human autism genes, suggesting that $5 \mathrm{hmC}$ may be influencing the observed autistic-like phenotype in these mice [79]. Since these findings were observed post-symptomatically in adult mice, it is unclear if the altered $5 \mathrm{hmC}$ represents a cause or a consequence of having autistic-like behaviors. Thus, these findings warrant a deeper investigation of this mouse model at earlier developmental time points.

Early life stressors have been associated with the development of mental illness, but the extent to which a gene $x$ environment interaction contributes to the development of autism has yet to be tested. However, prenatal stress reduced anxiety-like behaviors and altered gene expression in mice heterozygous for the 5-HTT gene [80]. Interestingly, these findings were more pronounced in female offspring, suggesting a gender-specific development of these altered behaviors. This study demonstrates a gene $x$ environment interaction and while this study did not examine $5 \mathrm{hmC}$, the $5 \mathrm{hmC}$-related data discussed in this review supports a $5 \mathrm{hmC}$ contribution to these altered behaviors and gene expression. Thus, it will be interesting to examine whether early life stress causes an autismprone individual (e.g., heterozygote for an autism risk allele) to develop full-blown autism phenotype, and if $5 \mathrm{hmC}$ plays a role in this process. If so, do these changes in $5 \mathrm{hmC}$ mirror those found in the homozygous mutants. This type of study could show that early life stress affects outcomes in offspring that may have a genetic predisposition to autism, a likely scenario for many mental disorders.

\section{Putative functions/clinical utilities of $5 \mathrm{hmC}$}

While many studies have shown that disruptions in $5 \mathrm{hmC}$ are linked with mental illness, the precise molecular function(s) of $5 \mathrm{hmC}$ remains unknown. Since $5 \mathrm{hmC}$ is generally associated with the transcription of neuronal genes, it likely has a role in the regulation of gene expression. Since $5 \mathrm{hmC}$ is enriched in post-mitotic neurons, it most likely has an impact on proper neurodevelopment by regulating expres- 
sion of genes responsible for neuronal propagation, development, and maintenance.

Several studies have revealed that altered $5 \mathrm{hmC}$ in differentially expressed genes is proximal to TF binding motifs $[29,55]$. Therefore, $5 \mathrm{hmC}$ may regulate $\mathrm{TF}$ binding to DNA, resulting in either up- or down-regulation of gene expression (Figure 1). $5 \mathrm{hmC}$ also has been associated with the regulation of alternative splicing of transcripts after cocaine-administration [31]. Here there was an overall greater change in isoform transcript levels, compared with full-length transcript levels [81]. An increase in $5 \mathrm{hmC}$ also was found in splice sites associated with upregulated spliced isoforms and a decrease of $5 \mathrm{hmC}$ in splice sites associated with downregulated spliced isoforms; hence, again coupling $5 \mathrm{hmC}$ changes in the brain with alternative splicing (Figure 1) [31]. Together, these studies implicate $5 \mathrm{hmC}$ in the regulation of transcriptional abundance and diversity.
$5 \mathrm{hmC}$ may also affect the binding of miRNAs (miRs). We recently discovered that altered $5 \mathrm{hmC}$ in differentially expressed genes is proximal to sequences that are complementary to seed regions of several miRs [Madrid, Alisch, Unpublished Data]. While miRs are generally thought to bind to mRNA and not DNA, $5 \mathrm{hmC}$ may have a co-transcriptional function in attracting or repelling miRs as the DNA is being transcribed into RNA (Figure 1). Interestingly, chronic variable stress on male mice alters miR content in sperm, and offspring from these sperm have a reduction in HPA axis stress responsivity [82]. Thus, miRs and $5 \mathrm{hmC}$ may work together in response to early life stressors by altering gene expression and physiological sensitivity to adversities, which may contribute to the origins of mental illnesses.

The instability of $5 \mathrm{hmC}$ levels following prenatal and/or acute stress underscores the potential for $5 \mathrm{hmC}$

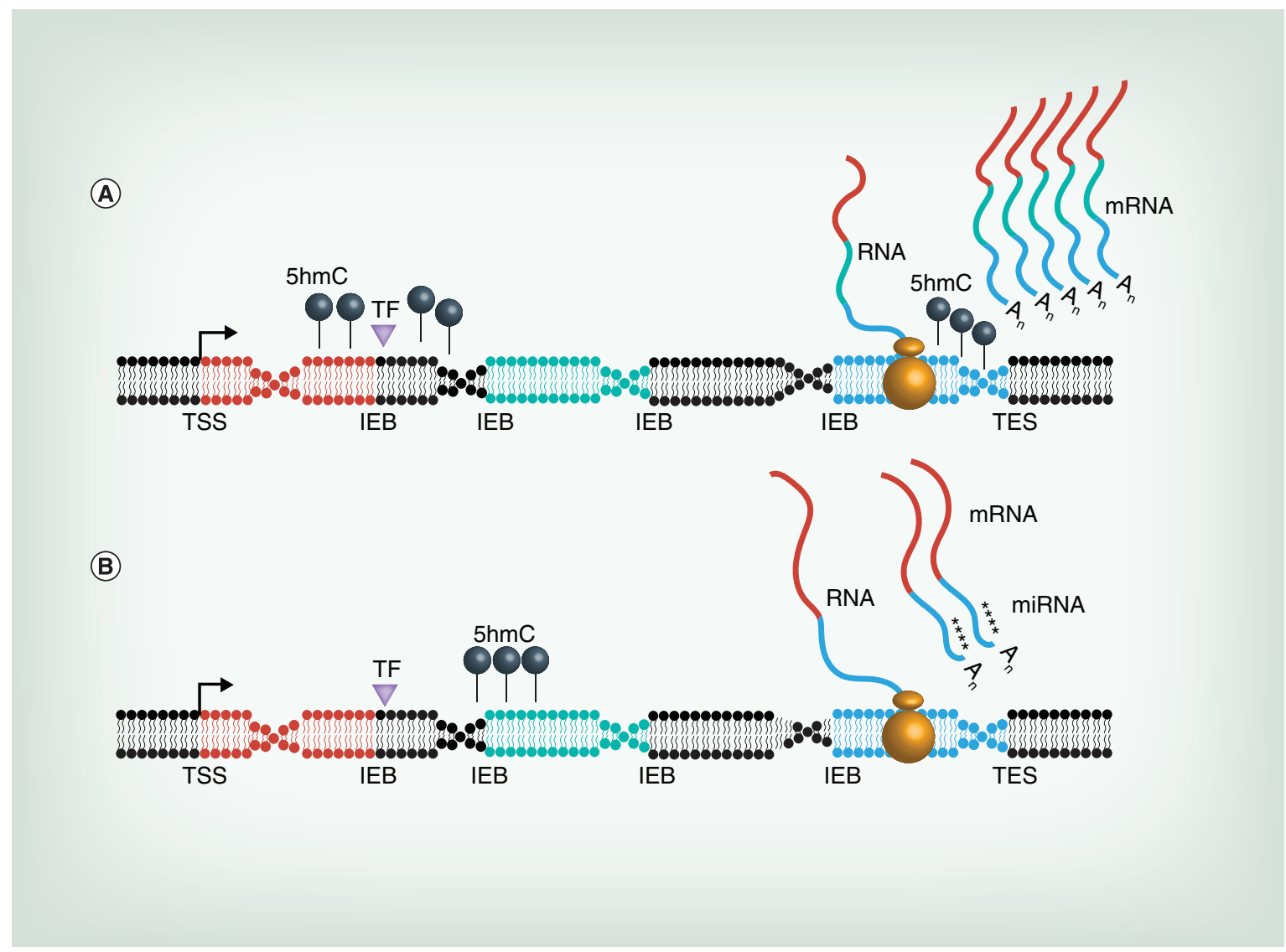

Figure 1. Schematic of putative functions of $5 \mathrm{hmC}$. When located near TF binding motifs (purple triangle), $5 \mathrm{hmC}$ (black lollipops) may regulate gene expression levels, resulting in either up- (A) or down- (B) regulation. When located at IEB, 5hmC (black lollipops) may regulate alternative splicing of transcripts, resulting in a shift from full-length transcripts (A) to splice isoforms (B). Exons are colored red, green, and blue. Intergenic and intragenic regions are gray. When located near sequences complementary to miRNAs seed regions, $5 \mathrm{hmC}$ (black lollipops) may have a latent function in repelling (A) or attracting (B) miRNAs as the DNA is being transcribed into RNA. Group of four asterisks represent miRs. Together, these studies implicate $5 \mathrm{hmC}$ in the regulation of transcriptional and translational abundance and diversity.

5hmC: 5-hydroxymethylcytosine; IEB: Intron/exon boundaries; TES: Transcription end site; TF: Transcription factor; TSS: Transcription start site. 
as a novel biomarker in the diagnosis of mental health. In addition, the presence/absence of these epigenetic modifications is reversible; thus, they may become relevant in therapeutic interventions [83], especially if methods to selectively modulate $5 \mathrm{hmC}$ in vivo are developed at the nucleotide level. Finally, $5 \mathrm{hmC}$ has gender-specific profiles, which is of interest because the development of several psychiatric disorders are seemingly gender-specific. For example, females show an increased risk in developing anxiety and depression while males show a disposition to development of autism and attention deficit hyperactivity disorder $[84,85]$. A recent study found gender-specific $5 \mathrm{hmC}$ on genes with ontological terms correlating with organ morphogenesis, system development and development of anatomical structures [30], suggesting that $5 \mathrm{hmC}$ may differentially influence the development of organs (e.g., the brain) among the genders. Together, these factors must be considered for clinical application and treatment endeavors.

In reality, our understanding of $5 \mathrm{hmC}$ functions is obscure and in its infancy. These molecular roles are likely to contribute to a broad spectrum of cellular functions from lifelong neurogenesis to cell death. Environmentally sensitive molecular mechanisms, such as $5 \mathrm{hmC}$, in the brain have become a significant focus of neuroscience research because of growing evidence that they are critical to the development of psychiatric disorders. Thus, in the coming years it will be of great interest to unravel these molecular functions of $5 \mathrm{hmC}$ that are contributing to developmental brain disorders.

\section{Conclusion}

Many of the studies discussed here suggest that mental illness is associated with disruptions in $5 \mathrm{hmC}$ throughout the genome. Since $5 \mathrm{hmC}$ is present embryonically and accumulates throughout life, primarily on genes associated with development, these studies support a role for $5 \mathrm{hmC}$ in developmental brain disorders. In addition, finding that $5 \mathrm{hmC}$ levels also can rapidly change at certain loci to reflect environmental stimuli and that these changes also can become stable, opens new perspectives in the study of epigenetic mechanisms underlying mental health and the origins of mental illness. The growing number of links between $5 \mathrm{hmC}$ and psychiatry disorders leads many to suspect a functional role for $5 \mathrm{hmC}$ in mental health. While it is still unknown whether these mental illness-related disruptions in $5 \mathrm{hmC}$ are causative or correlative to the outcome, defining the molecular function(s) of $5 \mathrm{hmC}$ will likely reveal the clinical relevance and should lead to the development of new, more specific drug therapies with more personalized therapeutic options for those with mental illness. Together, these studies highlight the emerging role of $5 \mathrm{hmC}$ in mental health and disease and bring new hope to the field of psychiatry.

\section{Future perspective}

This review has highlighted the new hope that the study of $5 \mathrm{hmC}$ brings to psychiatry. While traditional molecular genetics has been limited in revealing the etiology of mental illness, $5 \mathrm{hmC}$ may provide a viable molecular mechanism contributing to the onset and progression of such disorders and diseases. A more comprehensive understanding of $5 \mathrm{hmC}$, both functionally and as a biomarker, may provide a more objective measurement toward a more precise diagnosis of patients with mental illness. Ultimately, the development of methods to modulate $5 \mathrm{hmC}$ at the nucleotide level in genes contributing to mental illness may direct pharmaceutical companies in more fruitful directions of drug development for the treatment of mental illness. One such approach that might help achieve this nucleotide-specific modulation of $5 \mathrm{hmC}$ within the genome is to tether clustered, regularly interspaced short palindromic repeats (CRISPR) segments of DNA with either DNA methyltransferases or TET enzymes to selectively methylate or hydroxymethylate specific regions of the genome, respectively. Such a method would allow for specific modulation of $5 \mathrm{hmC}$ on genes that are known to have altered of $5 \mathrm{hmC}$ in mental illnesses, potentially allowing for personalized treatment and prevention of psychiatric disorders.

$5 \mathrm{hmC}$ in the context of mental illness is new and rapidly growing, suggesting that the future of such research is bright and prospectively fruitful. Our group expects that in the coming years disruptions of $5 \mathrm{hmC}$ will be examined in a cell-specific fashion, allowing researchers to study $5 \mathrm{hmC}$ at a much higher resolution. Future cell-specific examinations of genome-wide $5 \mathrm{hmC}$ in specific neural circuits that contribute to behavioral outcomes will provide an objective marker for a much quicker more reliable diagnosis that will lead to personalized treatments for those at high risk of developing a full-blown mental disorder.

\section{Disclaimer}

Any opinions, findings, and conclusions or recommendations expressed in this material are those of the author(s) and do not necessarily reflect the views of the National Science Foundation.

\section{Financial \& competing interests disclosure}

This work was supported in part by National Science Foundation under Grant No. 1400815 (A Madrid), NARSAD Young Investigator Grant from the Brain \& Behavioral Research Foundation \#22669 (LA Papale), the University of Wisconsin- 
Madison Department of Psychiatry, University of Wisconsin Vilas Life Cycle Professorships \#133AAA2989, University of Wisconsin Graduate School \#MSN184352 (all to RS Alisch). The authors have no other relevant affiliations or financial involvement with any organization or entity with a financial interest in or financial conflict with the subject matter or materials discussed in the manuscript apart from those disclosed.
No writing assistance was utilized in the production of this manuscript.

\section{Open Access}

This work is licensed under the Attribution-NonCommercialNoDerivatives 4.0 Unported License. To view a copy of this license, visit http://creativecommons.org/licenses/by-nc-nd/4.0/

Executive summary

State of psychiatry

- Diagnosis is based on subjective measures that are primarily acquired through behavioral assessments.

- Genetic influences often sought to explain the etiology of mental health.

- The broad heritability and heterogeneity of mental illness has limited the success of molecular genetics to determine its etiology.

- Crucial molecular mechanisms that cause variations in the expression of key genes in the brain are currently ignored by molecular psychiatry, which is contributing to misdiagnoses and ineffective treatments: a fundamental problem in the field of psychiatry.

Epigenetics \& 5-hydroxymethylcytosine

- Environmentally sensitive modifications that are heritable and often induce stable changes in gene expression.

- 5-hydroxymethylcytosine $(5 \mathrm{hmC})$ is a recently identified modification of cytosine that shows significant enrichment in brain over peripheral tissues and on genes known to have neuronal and synaptic functions.

- $5 \mathrm{hmC}$ is present embryonically and accumulates throughout life, suggesting a potential lifelong role in development.

- The presence/absence of $5 \mathrm{hmC}$ modifications are reversible.

$5 \mathrm{hmC}$ in mental health

- $5 \mathrm{hmC}$ disruptions have been associated with the development of early and late onset mental illnesses such as autism and Alzheimer's disease.

- Although linked to mental health, disruptions of $5 \mathrm{hmC}$ are not currently known to be causative or correlative of mental stability.

- Function(s) of $5 \mathrm{hmC}$ remain elusive, warranting deeper investigations aimed at unlocking the full potential of $5 \mathrm{hmC}$ as a therapeutic measure of mental health.

- Potential biomarker in the personalized diagnosis of mental illness.

Potential functions of $5 \mathrm{hmC}$

- Current understanding of $5 \mathrm{hmC}$ function(s) is obscure and in its infancy.

- Alterations of gene expression may be through transcription factor binding, miRNA binding and/or alternative splicing of transcripts to generate isoforms.

- Gender-specific distributions, which may explain gender-specific dispositions to mental illness (e.g., depression in females and autism in males).

Future perspective

- The development of personalized therapeutic manipulations of $5 \mathrm{hmC}$ to prevent onset or progression of mental illness through methods such as CRISPR to target specific genomic loci.

- The development of cell-specific profiling of specific neural circuits that contribute to behavioral outcomes will provide an objective marker for a much quicker and more reliable diagnosis that will lead to personalized treatments for those at high risk of developing a full-blown mental illness.

- The study of $5 \mathrm{hmC}$ brings new hope to the field of psychiatry through its promising clinical relevance.

\section{References}

Papers of special note have been highlighted as:

- of interest; $\bullet$ of considerable interest

1 Bird A. DNA methylation patterns and epigenetic memory. Genes Dev. 16(1), 6-21 (2002).

2 Sharma RP, Gavin DP, Grayson DR. CpG methylation in neurons: message, memory, or mask? Neuropsychopharmacol. 35(10), 2009-2020 (2010).

3 Han JA, An J, Ko M. Functions of TET proteins in hematopoietic transformation. Mol. Cells 38(11), 925-935 (2015).
4 Suzuki MM, Bird A. DNA methylation landscapes: provocative insights from epigenomics. Nat. Rev. Genet. 9(6), 465-476 (2008).

5 Robertson KD. DNA methylation and human disease. Nat. Rev. Genet. 6(8), 597-610 (2005).

6 Abdolmaleky HM, Cheng KH, Faraone SV et al. Hypomethylation of MB-COMT promoter is a major risk factor for schizophrenia and bipolar disorder. Hum. Mol. Genet. 15(21), 3132-3145 (2006).

7 Poulter MO, Du L, Weaver IC et al. GABAA receptor promoter hypermethylation in suicide brain: implications 
for the involvement of epigenetic processes. Biol. Psychiatry 64(8), 645-652 (2008).

8 Kuratomi G, Iwamoto K, Bundo $\mathrm{M}$ et al. Aberrant DNA methylation associated with bipolar disorder identified from discordant monozygotic twins. Mol. Psychiatry 13(4), 429-441 (2008).

9 Kappeler L, Meaney MJ. Epigenetics and parental effects. Bioessays 32(9), 818-827 (2010).

10 Weaver IC, Cervoni N, Champagne FA et al. Epigenetic programming by maternal behavior. Nat. Neurosci. 7(8), 847-854 (2004).

11 Wu H, Zhang Y. Mechanisms and functions of Tet proteinmediated 5-methylcytosine oxidation. Genes Dev. 25(23), 2436-2452 (2011).

12 Penn NW, Suwalski R, O’Riley C, Bojanowski K, Yura $\mathrm{R}$. The presence of 5-hydroxymethylcytosine in animal deoxyribonucleic acid. Biochem. J. 126(4), 781-790 (1972).

13 Kriaucionis S, Heintz N. The nuclear DNA base 5-hydroxymethylcytosine is present in Purkinje neurons and the brain. Science 324(5929), 929-930 (2009).

- This paper is of considerable interest as it is one of the first to rediscover 5-hydroxymethylcytosine $(5 \mathrm{hmC})$ and find enrichment of $5 \mathrm{hmC}$ in mammalian brain tissue.

14 Sun W, Zang L, Shu Q, Li X. From development to diseases: the role of $5 \mathrm{hmC}$ in brain. Genomics 104(5), 347-351 (2014).

15 Wang J, Tang J, Lai M, Zhang H. 5-hydroxymethylcytosine and disease. Mutat. Res. Rev. Mutat. Res. 762 167-175 (2014).

16 He YF, Li BZ, Li Z et al. Tet-mediated formation of 5-carboxylcytosine and its excision by TDG in mammalian DNA. Science 333(6047), 1303-1307 (2011).

17 Ito S, D'Alessio AC, Taranova OV, Hong K, Sowers LC, Zhang Y. Role of Tet proteins in $5 \mathrm{mC}$ to $5 \mathrm{hmC}$ conversion, ES-cell self-renewal and inner cell mass specification. Nature 466(7310), 1129-1133 (2010).

18 Kraus TF, Kilinc S, Steinmaurer M, Stieglitz M, Guibourt V, Kretzschmar HA. Profiling of methylation and demethylation pathways during brain development and ageing. J. Neural. Transm. (Vienna) 123(3), 189-203 (2016).

19 Cheng X. Structure and function of DNA methyltransferases. Annu. Rev. Biophys. Biomol. Struct. 24, 293-318 (1995).

20 Okano M, Bell DW, Haber DA, Li E. DNA methyltransferases Dnmt3a and Dnmt3b are essential for de novo methylation and mammalian development. Cell 99, 247-257 (1999).

21 Sharp AJ, Stathaki E, Migliavacca E et al. DNA methylation profiles of human active and inactive $\mathrm{X}$ chromosomes. Genome Res. 21, 1592-1600 (2011).

22 Ioshikhes IP, Zhang MQ. Large-scale human promoter mapping using CpG islands. Nat. Genet. 26(1), 61-63 (2000).

23 Irier HA, Jin P. Dynamics of DNA methylation in aging and Alzheimer's disease. CellBiol. 31(Suppl. 1), S42-S48 (2012).
24 Frommer M, McDonald LE, Millar DS et al. A genomic sequencing protocol that yields a positive display of 5-methylcytosine residues in individual DNA strands. Proc. Natl Acad. Sci. USA 89(5), 1827-1831 (1992).

25 Song CX, Szulwach KE, Fu Y et al. Selective chemical labeling reveals the genome-wide distribution of 5-hydroxymethylcytosine. Nat. Biotechnol. 29(1), 68-72 (2011).

26 Branco MR, Ficz G, Reik W. Uncovering the role of 5-hydroxymethylcytosine in theepigenome. Nat. Rev. Genet. 13, 7-13 (2012).

27 Chopra P, Papale LA, White AT et al. Array-based assay detects genome-wide $5-\mathrm{mC}$ and $5-\mathrm{hmC}$ in the brains of humans, non-human primates, and mice. BMC Genomics 15, 131 (2014).

28 Khare T, Pai S, Koncevicius K et al. 5-hmC in the brain is abundant in synaptic genes and shows differences at the exon-intron boundary. Nat. Struct. Mol. Biol. 19(10), 1037-1043 (2012).

29 Li S, Papale LA, Zhang Q et al. Genome-wide alterations in hippocampal 5-hydroxymethylcytosine links plasticity genes to acute stress. Neurobiol. Dis. 86, 99-108 (2016).

30 Gross JA, Pacis A, Chen GG et al. Characterizing 5-hydroxymethylcytosine in human prefrontal cortex at single base resolution. BMC Genomics 16, 672 (2015).

31 Feng J, Shao N, Szulwach KE et al. Role of Tet1 and 5 -hydroxymethylcytosine in cocaine action. Nat. Neurosci. 18(4), 536-544 (2015).

32 Yu M, Hon GC, Szulwach KE et al. Tet-assisted bisulfite sequencing of 5-hydroxymethylcytosine. Nat. Protoc. 7(12), 2159-2170 (2012).

33 Phase IMA, Qiu R, Wu X et al. Dynamics of 5-hydroxymethylcytosine and chromatin marks in mammalian neurogenesis. Cell Rep. 3(2), 291-300 (2013).

- This study is of interest as it shows an increase if $5 \mathrm{hmC}$ during neuronal differentiation in embryonic mouse brain.

34 Tan L, Xiong L, Xu W et al. Genome-wide comparison of DNA hydroxymethylation in mouse embryonic stem cells and neural progenitor cells by a new comparative hMeDIP-seq method. Nucleic Acids Res. 41(7), e84 (2013).

35 Kang J, Lienhard M, Pastor WA et al. Simultaneous deletion of the methylcytosine oxidases Tet 1 and Tet3 increases transcriptome variability in early embryogenesis. Proc. Natl Acad. Sci. USA 112(31), E4236-E4245 (2015).

36 Szulwach KE, Li X, Li Y et al. 5-hmC-mediated epigenetic dynamics during postnatal neurodevelopment and aging. Nat. Neurosci. 14(12), 1607-1616 (2011).

- This paper is of considerable interest as they display the stable and dynamic nature of $5 \mathrm{hmC}$ acquisition.

37 Chen H, Dzitoyeva S, Manev H. Effect of aging on 5-hydroxymethylcytosine in the mouse hippocampus. Restor. Neurol. Neurosci. 30(3), 237-245 (2012).

38 Zampieri M, Ciccarone F, Calabrese R, Franceschi C, Burkle A, Caiafa P. Reconfiguration of DNA methylation in aging. Mech. Ageing Dev. 151, 60-70 (2015). 
39 Chouliaras L, Van Den Hove DL, Kenis G et al. Agerelated increase in levels of 5-hydroxymethylcytosine in mouse hippocampus is prevented by caloric restriction. Curr. Alzheimer Res. 9(5), 536-544 (2012).

40 Al-Mahdawi S, Virmouni SA, Pook MA. The emerging role of 5-hydroxymethylcytosine in neurodegenerative diseases. Front. Neurosci. 8, 397 (2014).

41 Chouliaras L, Mastroeni D, Delvaux E et al. Consistent decrease in global DNA methylation and hydroxymethylation in the hippocampus of Alzheimer's disease patients. Neurobiol. Aging 34(9), 2091-2099 (2013)

42 Condliffe D, Wong A, Troakes C et al. Cross-region reduction in 5-hydroxymethylcytosine in Alzheimer's disease brain. Neurobiol. Aging 35(8), 1850-1854 (2014).

43 Coppieters N, Dieriks BV, Lill C, Faull RL, Curtis MA, Dragunow M. Global changes in DNA methylation and hydroxymethylation in Alzheimer's disease human brain. Neurobiol. Aging 35(6), 1334-1344 (2014).

44 Bradley-Whitman MA, Lovell MA. Epigenetic changes in the progression of Alzheimer's disease. Mech. Ageing Dev. 134(10), 486-495 (2013).

45 Lashley T, Gami P, Valizadeh N, Li A, Revesz T, Balazs R. Alterations in global DNA methylation and hydroxymethylation are not detected in Alzheimer's disease. Neuropathol. Appl. Neurobiol. 41(4), 497-506 (2015).

46 Walker FO. Huntington's disease. Lancet 369(9557), 218-228 (2007)

47 Lee J, Hwang YJ, Kim KY, Kowall NW, Ryu H. Epigenetic mechanisms of neurodegeneration in Huntington's disease. Neurotherapeutics 10(4), 664-676 (2013).

48 Reik W, Maher ER, Morrison PJ, Harding AE, Simpson SA. Age at onset in Huntington's disease and methylation at D4S95. J. Med. Genet. 30 (3), 185-188 (1993).

49 Wang F, Yang Y, Lin X et al. Genome-wide loss of 5-hmC is a novel epigenetic feature of Huntington's disease. Hum. Mol. Genet. 22(18), 3641-3653 (2013).

50 Villar-Menendez I, Blanch M, Tyebji S et al. Increased 5-methylcytosine and decreased 5-hydroxymethylcytosine levels are associated with reduced striatal A2AR levels in Huntington's disease. Neuromolecular Med. 15(2), 295-309 (2013).

51 McKinnon PJ. ATM and ataxia telangiectasia. EMBO Rep. 5(8), 772-776 (2004).

52 Jiang D, Zhang Y, Hart RP, Chen J, Herrup K, Li J. Alteration in 5-hydroxymethylcytosine-mediated epigenetic regulation leads to Purkinje cell vulnerability in ATM deficiency. Brain 138(Pt 12), 3520-3536 (2015).

- These findings are of interest as they link $5 \mathrm{hmC}$ deficits in human ataxia-telangiectasia and DNA damage signaling regulation.

53 Hagerman PJ, Hagerman RJ. Fragile X-associated tremor/ ataxia syndrome. Ann. NY Acad. Sci. 1338, 58-70 (2015).

54 Santoro MR, Bray SM, Warren ST. Molecular mechanisms of fragile X syndrome: a twenty-year perspective. Annu. Rev. Pathol. 7, 219-245 (2012).
55 Yao B, Lin L, Street RC et al. Genome-wide alteration of 5-hydroxymethylcytosine in a mouse model of fragile $\mathrm{X}$-associated tremor/ataxia syndrome. Hum. Mol. Genet. 23(4), 1095-1107 (2014).

56 Irier H, Street RC, Dave R et al. Environmental enrichment modulates 5-hydroxymethylcytosine dynamics in hippocampus. Genomics 104(5), 376-382 (2014).

57 Li S, Papale LA, Kintner DB et al. Hippocampal increase of 5 -hmC in the glucocorticoid receptor gene following acute stress. Behav. Brain Res. 286, 236-240 (2015).

58 Cirelli C, Faraguna U, Tononi G. Changes in brain gene expression after long-term sleep deprivation. J. Neurochem. 98(5), 1632-1645 (2006).

59 Li X, Wei W, Zhao QY et al. Neocortical Tet3-mediated accumulation of 5-hydroxymethylcytosine promotes rapid behavioral adaptation. Proc. Natl Acad. Sci. USA 111(19), 7120-7125 (2014).

60 Rudenko A, Dawlaty MM, Seo J et al. Tet1 is critical for neuronal activity-regulated gene expression and memory extinction. Neuron 79(6), 1109-1122 (2013).

- This paper is of interest as it suggests an epigenetic role in the pathogenesis of neuropsychiatric disorders such as depression.

61 Roth TL, Sweatt JD. Epigenetic marking of the BDNF gene by early-life adverse experiences. Horm. Behav. 59(3), 315-320 (2011).

62 Labonte B, Suderman M, Maussion G et al. Genomewide epigenetic regulation by early-life trauma. Arch. Gen. Psychiatry 69 (7), 722-731 (2012).

63 McGowan PO, Sasaki A, D’Alessio AC et al. Epigenetic regulation of the glucocorticoid receptor in human brain associates with childhood abuse. Nat. Neurosci. 12(3), 342-348 (2009).

64 Oberlander TF, Weinberg J, Papsdorf M, Grunau R, Misri $S$, Devlin AM. Prenatal exposure to maternal depression, neonatal methylation of human glucocorticoid receptor gene $(\mathrm{NR} 3 \mathrm{Cl})$ and infant cortisol stress responses. Epigenetics 3(2), 97-106 (2008).

65 Massart R, Suderman M, Provencal N et al. Hydroxymethylation and DNA methylation profiles in the prefrontal cortex of the non-human primate rhesus macaque and the impact of maternal deprivation on hydroxymethylation. Neuroscience 268 139-148 (2014).

66 Koenig JI, Elmer GI, Shepard PD et al. Prenatal exposure to a repeated variable stress paradigm elicits behavioral and neuroendocrinological changes in the adult offspring: potential relevance to schizophrenia. Behav. Brain Res. 156(2), 251-261 (2005).

67 Guidotti A, Dong E, Tueting P, Grayson DR. Modeling the molecular epigenetic profile of psychosis in prenatally stressed mice. Prog. Mol. Biol. Transl. Sci. 128, 89-101 (2014).

68 Matrisciano F, Tueting P, Dalal I et al. Epigenetic modifications of GABAergic interneurons are associated with the schizophrenia-like phenotype induced by prenatal stress in mice. Neuropharmacol. 68, 184-194 (2013).

69 Brown AS. The environment and susceptibility to schizophrenia. Prog. Neurobiol. 93(1), 23-58 (2011). 
70 Dong E, Gavin DP, Chen Y, Davis J. Upregulation of TET1 and downregulation of $A P O B E C 3 A$ and $A P O B E C 3 C$ in the parietal cortex of psychotic patients. Transl. Psychiatry 2, e159 (2012).

71 Tseng PT, Lin PY, Lee Y et al. Age-associated decrease in global DNA methylation in patients with major depression. Neuropsychiatr. Dis. Treat. 10, 2105-2114 (2014).

72 Gaugler T, Klei L, Sanders SJ et al. Most genetic risk for autism resides with common variation. Nat. Genet. 46(8), 881-885 (2014).

73 Bulik-Sullivan B, Finucane HK, Anttila V et al. An atlas of genetic correlations across human diseases and traits. Nat. Genet. 47(11), 1236-1241 (2015).

74 Gardener H, Spiegelman D, Buka SL. Prenatal risk factors for autism: comprehensive meta-analysis. Br. J. Psychiatry 195(1), 7-14 (2009).

75 Rogers EJ. Has enhanced folate status during pregnancy altered natural selection and possibly Autism prevalence? A closer look at a possible link. Med. Hypotheses 71(3), 406-410 (2008).

76 Wang T, Pan Q, Lin L et al. Genome-wide DNA hydroxymethylation changes are associated with neurodevelopmental genes in the developing human cerebellum. Hum. Mol. Genet. 21(26), 5500-5510 (2012).

77 Penagarikano O, Lazaro MT, Lu XH et al. Exogenous and evoked oxytocin restores social behavior in the Cntnap2 mouse model of autism. Sci. Transl. Med. 7(271), 271ra-278 (2015).

78 Penagarikano O, Abrahams BS, Herman EI et al. Absence of CNTNAP2 leads to epilepsy, neuronal migration abnormalities, and core autism-related deficits. Cell 147(1), 235-246 (2011).

79 Papale LA, Zhang Q, Li S, Chen K, Keles S, Alisch RS. Genome-wide disruption of 5-hydroxymethylcytosine in a mouse model of autism. Hum. Mol. Genet. 24(24), 7121-7131 (2015).

- This study is of interest as it formally links $5 \mathrm{hmC}$ alterations in the brain of a mouse model of autism, suggesting an epigenetic role (e.g., $5 \mathrm{hmC}$ ) in autism pathogenesis.

80 Van Den Hove DL, Jakob SB, Schraut KG et al. Differential effects of prenatal stress in 5-Htt deficient mice: towards molecular mechanisms of gene $\times$ environment interactions. PLoS ONE 6(8), e22715 (2011).

81 Feng J, Wilkinson M, Liu X et al. Chronic cocaine-regulated epigenomic changes in mouse nucleus accumbens. Genome Biol. 15(4), R65 (2014).

82 Rodgers AB, Morgan CP, Bronson SL, Revello S, Bale TL. Paternal stress exposure alters sperm microRNA content and reprograms offspring HPA stress axis regulation. J. Neurosci. 33(21), 9003-9012 (2013).

83 Szyf M. Epigenetics, a key for unlocking complex CNS disorders? Therapeutic implications. Eur. Neuropsychopharmacol. 25(5), 682-702 (2015).

84 Nolen-Hoeksema S. Sex differences in unipolar depression: evidence and theory. Psychol. Bull. 101(2), 259-282 (1987).

85 Wooten GF, Currie LJ, Bovbjerg VE, Lee JK, Patrie J. Are men at greater risk for Parkinson's disease than women? J. Neurol. Neurosurg. Psychiatry 75(4), 637-639 (2004). 